\title{
User Analysis Mechanisms based Mobile Fitness System
}

\author{
Jong Won Lee, Han Kil Kim, Hoe Kyung Jung
}

Department of Computer Engineering, Paichai University, Korea

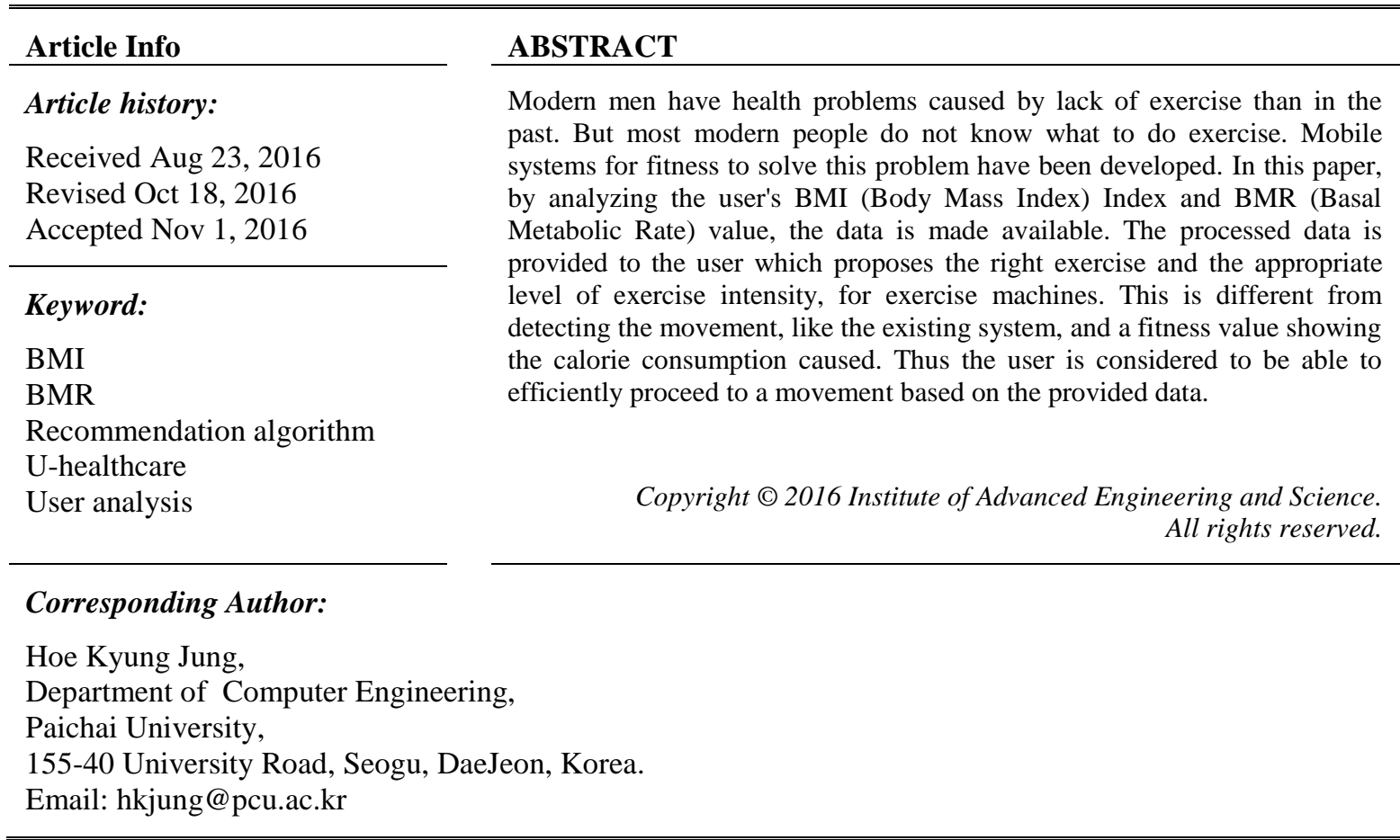

\section{INTRODUCTION}

In the modern society, obesity has been increasing due to a number of reasons, like improper food intakes and lack of exercise. Lack of muscle mass induces various diseases. As a result, the importance of strength training is increasing day by day. However, strength training should be conducted mostly through a weight training room, and there was no way to determine quantity of exercise. To solve this problem, various fitness systems have been developed [1-3]. Existing fitness systems sense the motion of the user and inform the consumed calorie value based on the sensor of a mobile device. However, the data provided to existing systems are not high accuracy. Also it notifes only the bumed calories value, and information on recommendations for strength training is insufficient. In another method the system can analyze the user's body information, and provide the data to indicate how the exercise and use the fitness equipment. In this way it is difficult for the system to provide a generic data analysis, because only the information of individual users. There are also other problems with the information that is available and the user needs to actually [4-7].

In this paper, we develop a system that can be used indoor and outdoor, to manage fitness due to lack of strength exercise. Mechanisms of the system analysis the users information and provide the processed data. It also provides three different recommendation data, based on different algorithm in order to solve the shortcomings of the existing systems of not providing fitness data required by the user.

Recommendation algorithm is made based on the user's BMI index, BMR value and exercise level, etc. through which the user is able to receive the correct data than other fitness system. In addition, group of users with similar levels of BMI index as the user, notifies info and exercise machines with users belonging to the same group as the users. Because of this it is possible to proceed with the strength training based on various informations. Also we have used the inductive reasoning method, which allows reflecting the choice of the user to provide the data. 


\section{DESIGN}

This describes the design of our fitness system.

\subsection{System Design}

Figure 1 configuration of the system and Figure 2 Flow chart of the system.

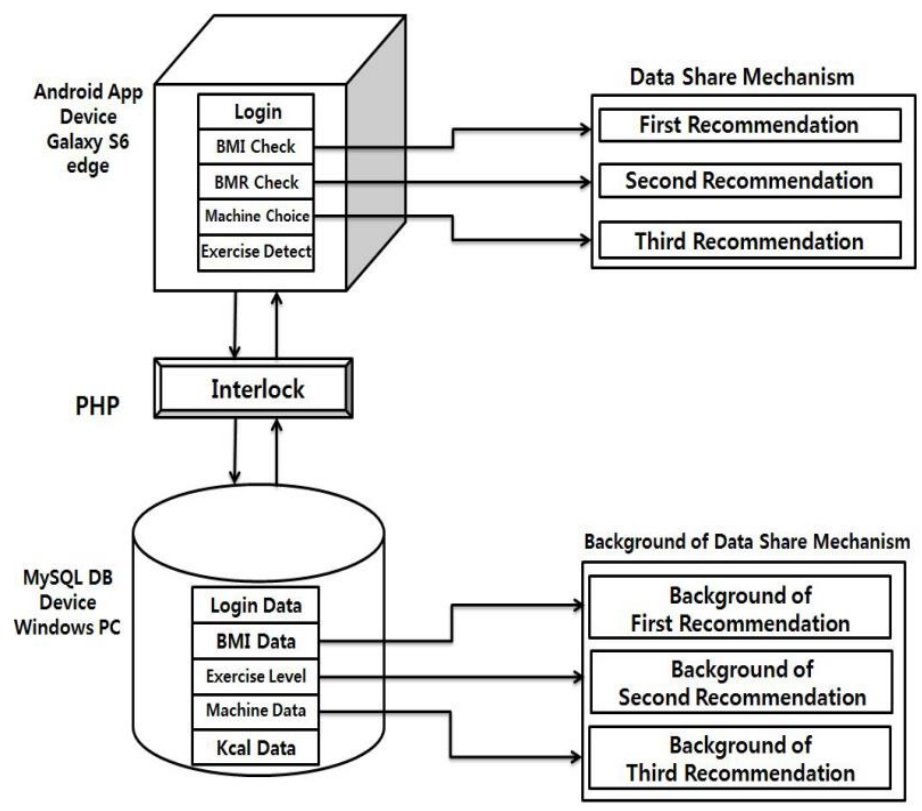

Figure 1. System Configuration

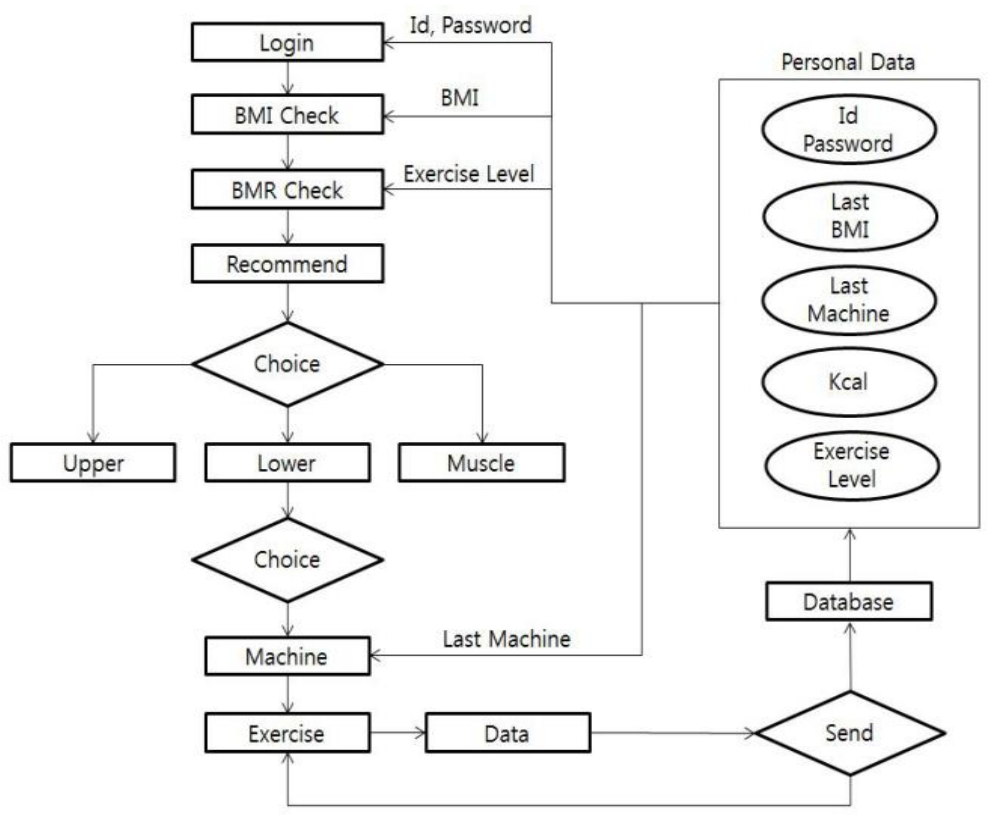

Figure 2. System Processing

Configuration of the system consists of android mobile, PHP, and database in pc. The main function of the application are as follows, application login, BMI index check, BMR value check, sports equipment selection, motion detection and the three of recommendation algorithms. PHP is linked to a 
database between android applications and server pc. The database stores the login data, BMI data, BMR data, exercise equipment data, calorie data available in android applications.

The flow of applications will begin by performing a login. Based on the physical information of the user input to determine the BMI index and BMR value check then the exercise equipment and exercise recommendations. The user will select the upper body exercise, lower body exercise, site-specific exercise. After selecting exercise machine when the user starts the exercise proximity sensor and the acceleration sensor senses the user's motion. The data is transmitted and stored once the user finishes the exercise. The stored data are analyzed by a recommendation algorithm. So it proceeds to the grouping according to the user's current BMI index. If the user selects the Upper, application indicate information to user. Waht other user in same BMI group used Upper body exercise machine. It also view toast messages to other users of exercise equipment in the similar BMI group to which they belong. This makes recommendation about whether the people of the BMI index, as the user generally uses exercise equipment.

\subsection{Recommendation Algorithm}

Figure 3 is a flow chart of the first recommendation algorithm fitness system.

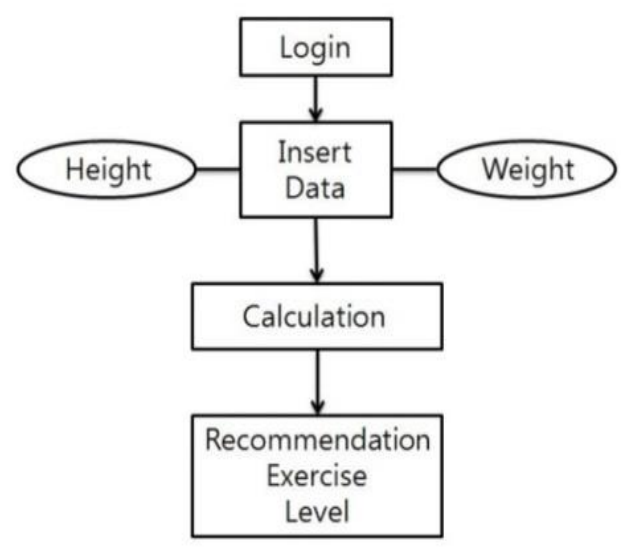

Figure 3. First Recommendation Algorithm

The first recommend algorithm computes the user's BMI index and recommends the Exercise machine of a suitable strength on the user's BMI index. Figure 4 is a flow diagram of a second recommendation algorithm.

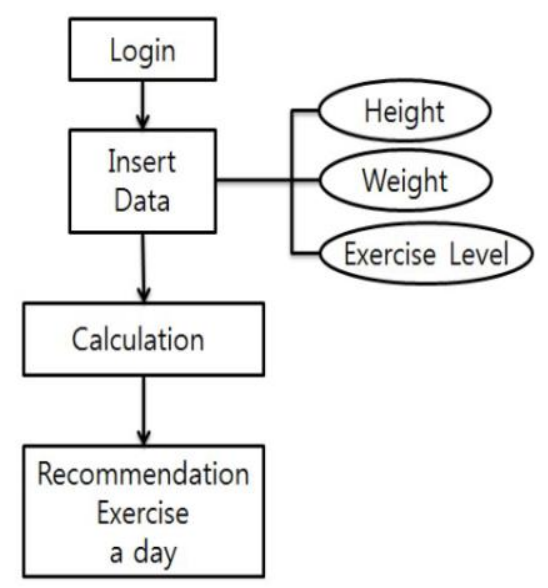

Figure 4. Second Recommendation Algorithm 
The second recommendation algorithm recommends appropriate amount of exercise as day goes by considering the BMR levels and exercise levels. Figure 5 is a flow diagram of a third recommendation algorithm. The third recommendation algorithm proceeds recommendations for trainers based on the data of the user's BMI and the BMI index group. It also specifies the muscles that enhance the value and calories that you consume through exercise.

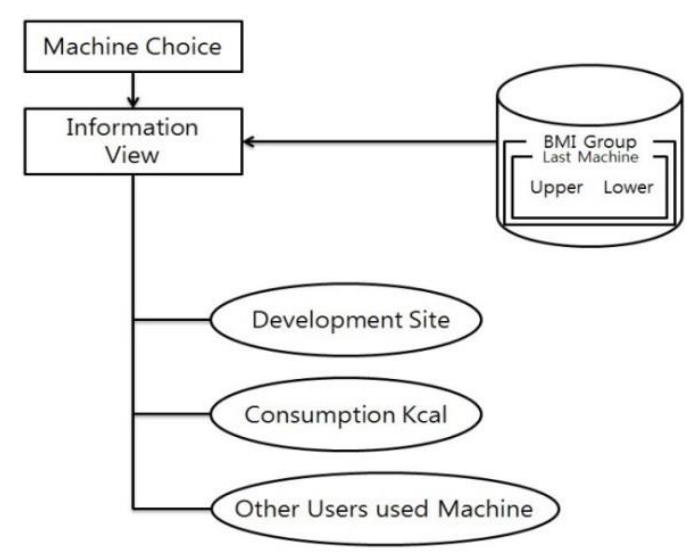

Figure 5. Third Recommendation Algorithm

\section{IMPLEMENT}

This chapter describes implemention of fitness system.

\subsection{Application Implement}

Figure 6 is a flow diagram of the Activity application.

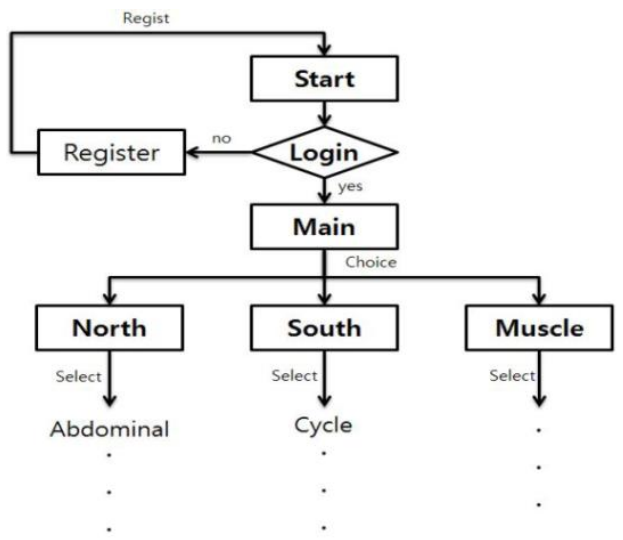

Figure 6. Application Activity Flow

Activity starts with the user's login and Register Activity performs new user registration. In the Main Activity input and analyzes the information to the user's body allows the strength of the recommended exercise. The body of the North Activity trainers, South Activity, Muscle Activity in the muscle of the lower body exercise equipment and exercise equipment to show the list in each Activity. Figure 7 is a flow chart of Main Activity. 


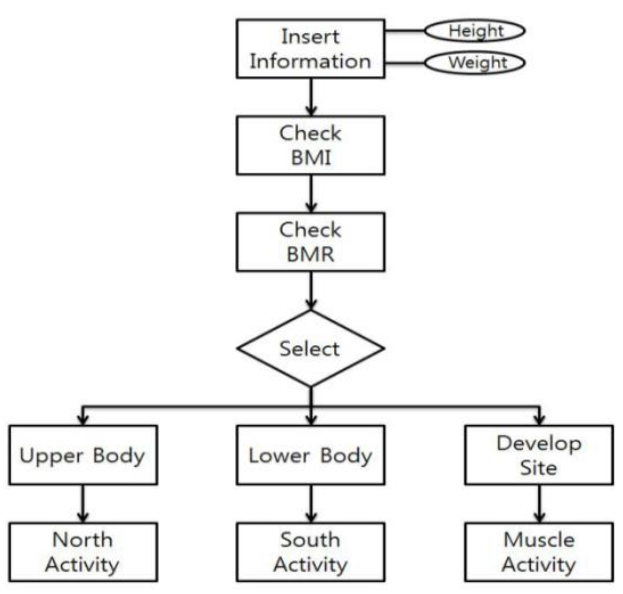

Figure 7. Application Main Activity Flow

The user must input their height and weight. If they do not enter one of the two, then they cannot proceed with the application. After entering the data, proceed to step Check BMI to show the user status as underweight, normal weight, overweight, obese, obesity, depending on your BMI Index. And it allows the user to recommend the appropriate exercise intensity. The intensity of exercise is represented by Level 1, 2, 3. Check BMR step shows the exercise basal metabolic rate based on the user's height and weight, the metabolic rate shown by analysis to be conducted in one day. And if you want an upper body exercise, press the North Body button, if you want a lower body exercise, press the button Lower Body. If you want to exercise for a specific part of the body there is press the button to Develop Site. Each button has lean athletic equipment, lower body exercise equipment, organized by the show muscle-specific exercise equipment in the list.

\subsection{Screen of Implement}

Figure 8 is a screen of the user to analyze the BMR value.

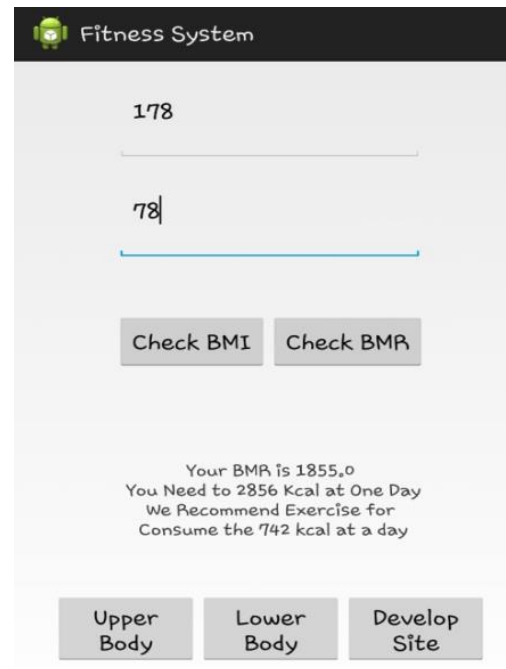

Figure 8. Screen of BMR Value Check

The user results are based on analyzing the user's body information BMR and metabolic rate, indicates the quantity of exercise suitable for the body. 


\subsection{Review}

Other fitness systems are mostly based on the body of the user by analyzing the information and not providing data for the exercise intensity and exercise. Also providing data related to exercise mechanism is not provided in them. This system was developed for fitness by keeping in mind the shortcommings of other fitness systems.

The first recommendation algorithm suggests suitable exercise intensity for the users' body. The second recommendation algorithm indicates the appropriate level of exercise which the user can proceeds in a day. The third recommendation algorithm informs the trainers of BMI group, such as the user. Thereby providing a common data based on the inductive reasoning methods.

Users appropriate exercise intensity is compared to other fitness systems on the basis of data provided by this system, the appropriate level of exercise, you can see exactly the right exercise equipment. Accordingly, it is possible to proceed with the effective strength training.

\section{CONCLUSION}

Most of the systems used in conventional fitness are based on motion sensors, and displaying consumed calorie value. It also provides data to the user, which is part of the data necessary to the user. To solve this problem, a fitness system that is currently being studied have been developed based on different algorithms, which recommendes the user on how to exercise or use the exercise equipment.

Proposed system is available recommend the appropriate data to the user by analyzing the information of user body. Analysis of user application mechanism is based on three recommendation algorithm. The first recommendation algorithm analyzes the user's body information, and like to calculate the exercise BMI index. The second recommendation algorithm analyzes the BMR value and metabolic activity of the user like metabolic rate and tells quantity of exercise. The third recommendation algorithm that tells the trainers belonging to the same BMI group, such as the user, and makes recommendations to other users using exercise equipment that fits their BMI index. This user may be provided the data for strength training. Then the data makes it possible to perform the efficient exercise for the user.

Future research needs to, verify the efficiency of the system on a variety of exercise experiments. And we shall study the algorithm to continuously analyze and manage the user.

\section{ACKNOWLEDGEMENTS}

This research was supported by Basic Science Research Program through the National Research Foundation of Korea (NRF) funded by the Ministry of Education (No. 2014R1A1A2059842). This work was supported by the research grant of Pai Chai University in 2016.

\section{REFERENCES}

[1] G. Y. Cho, "Research on a Method for Efficient u-Healthcare Data Transmission in M2M Environment," The Society of Digital policy \& Management, vol/issue: 12(4), pp. 251-257, 2014.

[2] S. W. Kim and S. C. Shim, "Development of Mobile Healthcare System Using ECG Measurement," The Korea Institute of Information, and Communication Engineering, vol/issue: 18(8), pp. 2008-2016, 2014.

[3] S. H. Kim, "Design and Implementation of Moment Measurement System Using a Triaxial Accelerometer," Korean Institute of Information Technology, vol/issue: 13(3), pp. 1-7, 2015.

[4] J. G. Lee, et al., "Smart Medical Technology Standards-based Mobile Health," Korea Information Science Society, vol/issue: 33(3), pp. 31-44, 2015

[5] E. J. Lee and H. S. Kim, "Implementation of the Healthcare System Using Smart TV," The Korean Institute of Information Technology, vol/issue: 12(10), pp. 191-199, 2014

[6] R. J. Conejar and H. K. Kim, "Design U-Healthcare Web Services System," International Journal of Software Engineering and Its Applications, vol/issue: 9(3), pp. 209-216, 2015.

[7] J. W. Lee, "Design of Kalman Filter to Estimate Heart Rate Variability from PPG Signal for Mobile Healthcare," Journal of Information and Communication Convergence Engineering, vol/issue: 8(2), pp. 201-204, 2010. 


\section{BIOGRAPHIES OF AUTHORS}

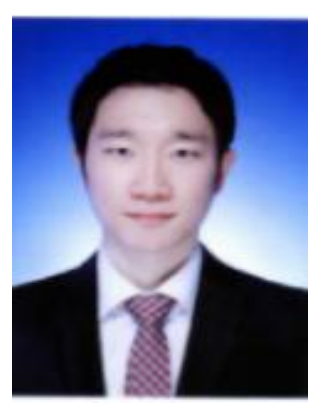

Jong Won Lee. He received the B.S., M.S. degree from the Department of Computer Engineering of Paichai University, Korea in 2014 and 2016. He is currently a Docorate course in Department of Computer Engineering of Paichai University. His current research interests include multimedia information processing, information retrieval system, and semantic web.

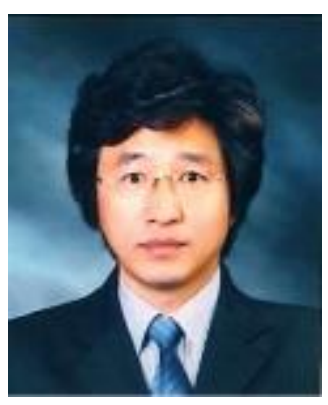

Han Kil Kim. He received the B.S., M.S. degrees from the Department of Electronic Engineering of Hanbat National University, Korea, in 2006, and 2011, and the Ph. D. degree in 2015 from the Department of Computer Engineering of Paichai University, Korea. Since 2006, he worked for the Department of Music \& Sound Technology at Korea University of Media Arts as a professor. His current research interests include information retrieval system, web 2.0, and the semantic web.

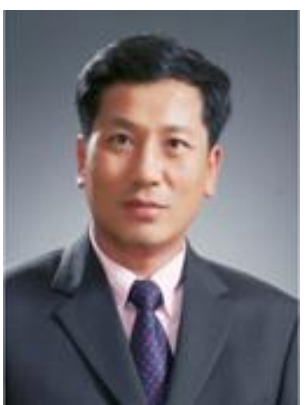

Hoe Kyung Jung. He received the M.S. degree in 1987 and Ph. D. degree in 1993 from the Department of Computer Engineering of Kwangwoon University, Korea. From 1994 to 1995, he worked for ETRI as a researcher. Since 1994, he has worked in the Department of Computer Engineering at Paichai University, where he now works as a professor. His current research interests include multimedia document architecture modeling, information processing, information retrieval, and databases. 